\title{
Correction to: Rehmanniae Radix Preparata suppresses bone loss and increases bone strength through interfering with canonical Wnt/ $\beta$-catenin signaling pathway in OVX rats
}

\author{
C. Liu ${ }^{1,2} \cdot$ L. Wang ${ }^{1,2} \cdot$ R. Zhu ${ }^{1} \cdot$ H. Liu ${ }^{1} \cdot$ R. Ma ${ }^{1,3} \cdot$ B. Chen ${ }^{1} \cdot$ L. Li $^{1} \cdot$ Y. Guo $^{1,4} \cdot$ Q. Jia ${ }^{1} \cdot$ S. Shi ${ }^{2} \cdot$ D. Zhao ${ }^{1} \cdot$ F. Mo ${ }^{1} \cdot$

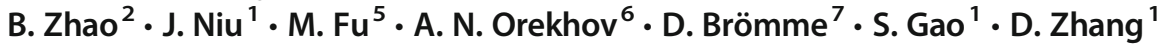

Published online: 18 June 2019

(C) International Osteoporosis Foundation and National Osteoporosis Foundation 2019

\section{Correction to: Osteoporos Int.}

https://doi.org/10.1007/s00198-018-4670-y

There was a mistake in the part of OVX rats model and $R R P$ intervention in the original publication. The dosage of $\mathrm{EV}(0.1 \mathrm{mg} / 1 \mathrm{~mL} / 100 \mathrm{~g})$ and RRP $(2.5 \mathrm{~g} / 1 \mathrm{~mL} / 100 \mathrm{~g})$ should be corrected as EV $(0.01 \mathrm{mg} / 1 \mathrm{~mL} / 100 \mathrm{~g})$ and RRP $(0.25 \mathrm{~g} /$ $1 \mathrm{~mL} / 100 \mathrm{~g}$ ).

D. Zhang

dongwei1006@gmail.com

1 Diabetes Research Center, Traditional Chinese Medicine School, Beijing University of Chinese Medicine, Beijing 100029, People's Republic of China

2 Chinese Materia Medica School, Beijing University of Chinese Medicine, Beijing 100029, China

3 Guang'anmen Hospital, China Academy of Chinese Medical Sciences, Beijing 100053, China
4 The Third Affiliated Clinical Hospital, Beijing University of Chinese Medicine, Beijing 100029, China

5 The Research Institute of McGill University Health Center, Montreal, Quebec H4A 3J1, Canada

6 Laboratory of Angiopathology, Russian Academy of Medical Sciences, Institute of General Pathology and Pathophysiology, Moscow 125315, Russia

7 Department of Oral Biological \& Medical Sciences, Faculty of Dentistry, The University of British Columbia, Vancouver, BC V6T 1Z3, Canada 
In addition, the names and serial numbers of compounds in Supplement Fig. 1 were unfortunately incorrect. The figure is reproduced here.

a

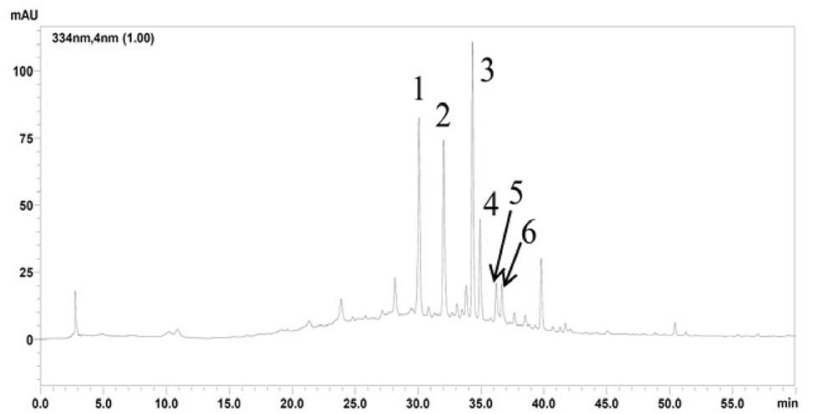

b

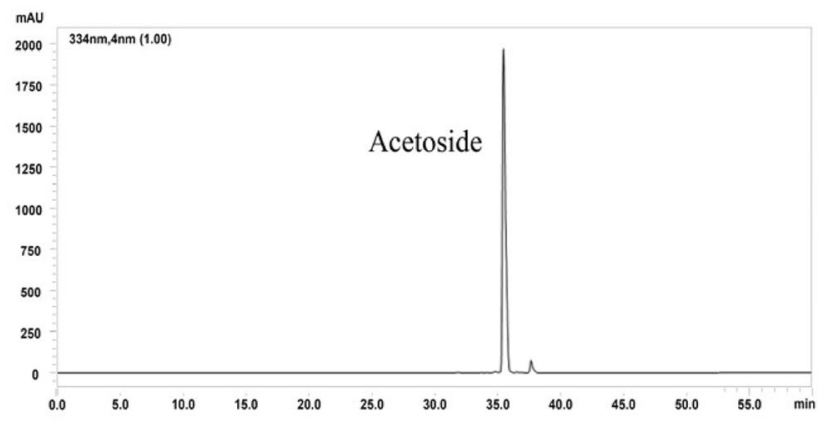

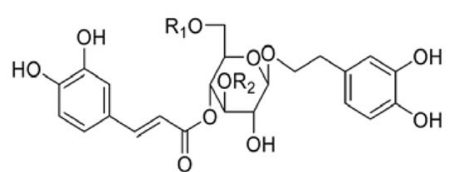

Echinacoside (1): $\mathrm{R}_{1}=$ Glc $\mathrm{R}_{2}=\mathrm{Rha}$

Acetoside (3): $\mathrm{R}_{1}=\mathrm{H} \quad \mathrm{R}_{2}=\mathrm{Glc}$

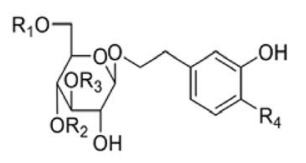

Jionoside A1/A2 (2):

$\mathrm{R}_{1}=$ Glc $\mathrm{R}_{2}=$ Feruloyl $\mathrm{R}_{3}=$ Rha $\mathrm{R}_{4}=\mathrm{OH}$

Jionoside B1/B2 (5)/ (6):

$\mathrm{R}_{1}=$ Glc $\mathrm{R}_{2}=$ Feruloyl $\mathrm{R}_{3}=$ Rha $\mathrm{R}_{4}=$ Ome

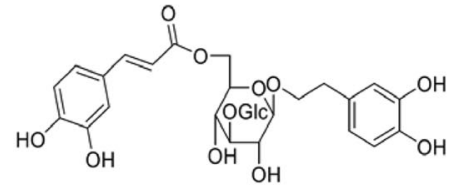

Isoacetoside (4)

Moreover, the authors carelessly uploaded the wrong images (Fig. 6a) for the Sham Group and OVX Group. The figure below shows the correct images. The error does not affect the interpretation and conclusion of the study. The 
$\mathbf{a}$

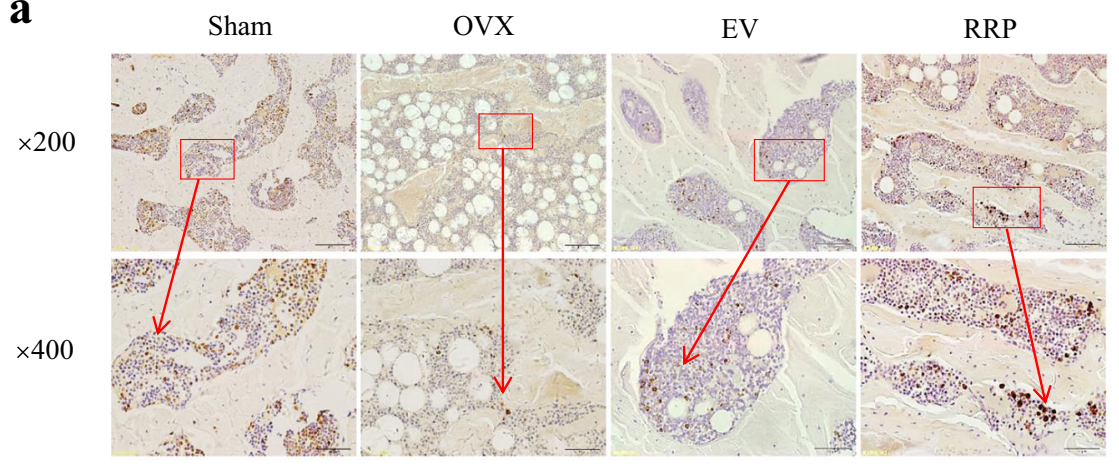

b

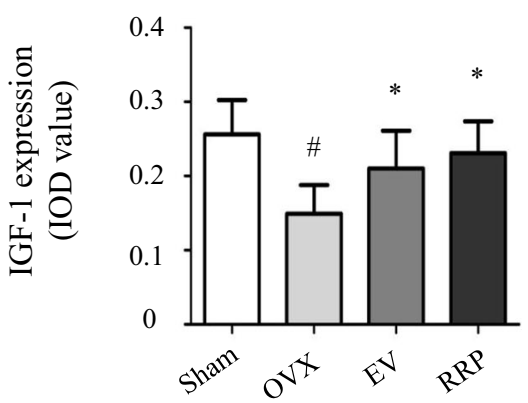

\section{c}

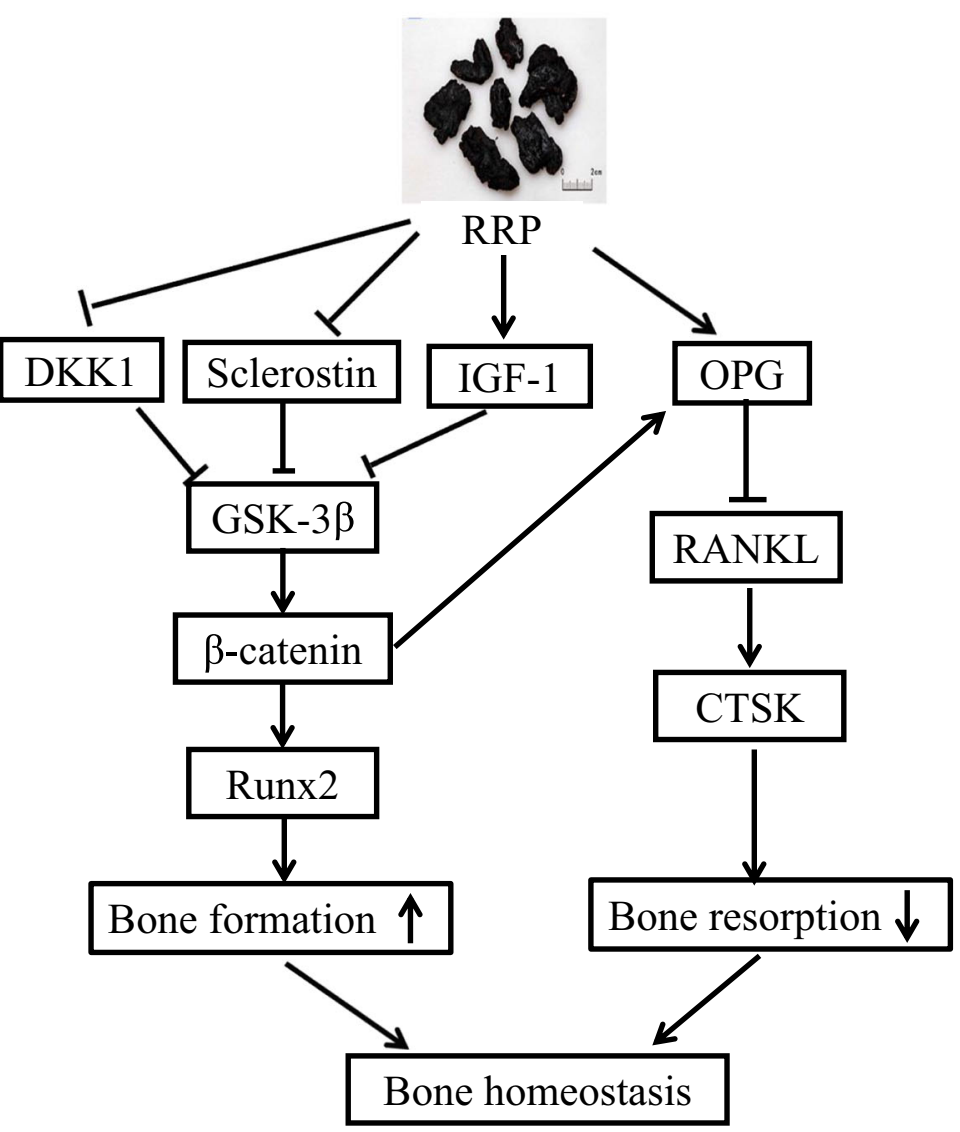

authors apologize for any inconvenience that this error may have caused.

Fig. 6 Rehmanniae Radix Preparata (RRP) improves IGF-1 expression in the femurs and potential mechanism of RRP prevents the development of osteoporosis in OVX rats. The representative micro-images (a) and their analyses (b) of immunohistochemical staining (sections were counterstained with hematoxylin; original magnification, $\times 200$ and $\times 400$ ) show the IGF-1 expression in the rat femurs of the treatment and control groups. Data are presented as mean $\pm \mathrm{SD}$. The dark brown particles denote positive staining. IOD denotes integrated optical density of interested areas. ${ }^{\#} p<0.05$ compared with Sham group, ${ }^{*} \mathrm{p}<0.05$ compared with OVX group. $n=$ 9. (c) The potential mechanism of Rehmanniae Radix Preparata (RRP) against osteoporosis in OVX rats. RRP may downregulate overexpression of DKK1 and sclerostin as well as increase IGF-1 expression, which further activates the $\mathrm{Wnt} / \beta$-catenin signaling pathway and contributes to subsequent bone formation. RRP 
may also increase OPG expression and secretion, which leads to an inhibition of the RANKL/cathepsin Kmediated bone resorption. In addition, the increased expression of $\beta$-catenin further activates OPG/RANKL signaling. The sign $(\downarrow)$ means promoting. The sign $(\perp)$ means inhibiting. CTSK, cathepsin K; DKK1, Dickkopfrelated protein-1; GSK-3 $\beta$, glycogen synthase kinase
$3 \beta$; IGF-1, insulin-like growth factor-1; OPG, osteoprotegerin; RANKL, receptor activator for nuclear factor- $\mathrm{KB}$ ligand

Publisher's note Springer Nature remains neutral with regard to jurisdictional claims in published maps and institutional affiliations. 